Investigaciones Fenomenológicas, vol. Monográfico 4/II (2013): Razón y vida, 279-293. e-ISSN: $1885-1088$

\title{
LA OBJETIVIDAD DE LOS VALORES EN HUSSERL Y SCHELER. UNA "DISPUTA FENOMENOLÓGICA"
}

\section{The ObJectivity of Values in Husserl AND Scheler. A "Phenomenological dispute"}

\author{
Mariana Chu García \\ Círculo Peruano de Fenomenología y Hermenéutica (CIphER)/ \\ Pontificia Universidad Católica del Perú, Perú \\ mchu@pucp.pe
}

\begin{abstract}
Resumen: De modo general, la posición de Husserl respecto de Scheler se puede resumir como el rechazo a una "metafísica ingenua". Del otro lado, la posición de este respecto del fundador de la fenomenología se puede caracterizar como desaprobación al "giro trascendental" y al privilegio del pensar sobre el sentir y valorar. Pese a estas diferencias irreconciliables, Husserl y Scheler coinciden no solo en la defensa de la objetividad de los valores. En este texto, quisiéramos delinear los trazos generales de lo que sería una disputa fenomenológica entre Husserl y Scheler respecto de la objetividad axiológica con el fin de mostrar cómo sus análisis coinciden y divergen.
\end{abstract}

Palabras clave: Husserl, Scheler, valores, fenomenología.

\begin{abstract}
In general terms, Husserl's position regarding Scheler can be summarized as the rejection of a "naive metaphysics". On the other hand, Scheler's position regarding the father of Phenomenology can be presented as the disapproval of the "transcendental turn" and of the priority of thought over feeling and valuing. In spite of these irreconcilable differences, Husserl and Scheler agree in the defence of the objectivity of values. In this paper, we will try to draw some general guidelines of what a phenomenological dispute between Husserl and Scheler concerning the objectivity of values would be like in order to show how theirs analyses coincide, and disagree.
\end{abstract}

Key Words: Husserl, Scheler, Values, Phenomenology.

En un texto titulado "Fenomenología y teoría del conocimiento", a propósito de la crítica de Wundt a las Investigaciones lógicas, Scheler se refiere a un equívoco común respecto de los libros de fenomenología: en ellos -se objeta-, nunca se dice qué son las cosas investigadas, sino solo lo que no son y, luego de una serie de negaciones, no se ofrece más que una tautología ${ }^{1}$. Quien lee las

\footnotetext{
${ }^{1}$ Scheler, Max, "Phänomenologie und Erkenntnistheorie", en Schriften aus dem Nachlass. Band I: Zur Ethik und Erkenntnislehre, Gesammelte Werke, vol. 10, Berna: Francke, 1957, p. 391 (en adelante, GW
} 
Investigaciones de este modo, responde Scheler, adopta una actitud equivocada, pues todas las negaciones y delimitaciones que se hacen en una exposición fenomenológica no son sino indicaciones que van guiando al lector hasta que la tautología le dice: "iAhora tienes que mirar, entonces lo verás!"². La fenomenología no fija definiciones, porque su objeto de conocimiento, las esencias y estructuras esenciales a priori, no es definible, sino solo experimentable o vivido de modo pleno en una intuición inmediata. A esta idea de una fenomenología que "se vive" o que solo adquiere sentido si uno mismo efectúa la reducción y la intuición eidética refiere la exigencia de "ir a las cosas mismas". Pero, dado que, si se trata de una esencia genuina, todos deben poder vivirla", no basta "ir a las cosas mismas": si los análisis fenomenológicos pretenden fundar algo, deben también mostrar cómo cualquiera puede tener por sí mismo la o las respectivas intuiciones eidéticas. Por eso, se ha planteado un imperativo para el fenomenólogo: "Decir lo visto y nada más que lo visto" ${ }^{4} \mathrm{o}$ "Si afirmas algo, muestra entonces cómo no importa quién podría acceder a la evidencia de lo que afirmas" ${ }^{5}$. ¿Qué pasa si el lector o interlocutor no llega a ver lo que se quiere mostrar? Se inicia lo que Scheler llama una "disputa" o "discusión fenomenológica" (phänomenologischer Streit), en la cual las palabras, y cualquier medio empleado, no son más que un modo de dar, en términos de Husserl, "material de intuición" (Anschauugsmaterial) para que cada uno "pueda descubrir el camino a sus propias evidencias intelectuales (Einsichten) sistemáticas" Para dirimir las discusiones fenomenológicas, que pueden tener distintas "explicaciones", no existe ningún criterio general ni a priori, hay que considerar caso por caso, dice Scheler ${ }^{7}$.

Nosotros quisiéramos ocuparnos aquí del caso de la teoría de valores con el fin de mostrar en qué sentido los análisis de Husserl y Scheler coinciden, y en qué radica su desacuerdo. Ahora bien, un diálogo real entre ellos nunca existió.

10); "Fenomenología y gnoseología", en La esencia de la filosofía y la condición moral del conocer, Buenos Aires: Editorial Nova, 1962, p. 77 (en adelante, FG).

${ }^{2}$ GW 10, p. 392; FG, p. 78.

${ }^{3}$ Ibid., p. 393; ibid., p. 81.

${ }^{4} \mathrm{Cf}$. Benoist, Jocelyn, "Il n'y a pas certes pas de phénoménologie, mais il y a bel et bien des problèmes phénoménologiques", en: Rue Descartes, n² 29 (2000), pp. 65-78.

${ }^{5}$ De Monticelli, Roberta, L'avenir de la phénoménologie. Méditations sur la connaissance personnelle, París: Aubier, 2000, p. 59.

6 Einleitung in der Ethik. Vorlesungen Sommersemester 1920 und 1924, ed. Henning Peucker, Dordrecht/Boston/Londres: Kluwer, Husserliana, tomo XXXVII, 2004, § 6, p. 3 (en adelante, Hua XXXVII).

${ }^{7}$ Cf. GW 10, pp. 393-394; FG, p. 81. 
Más allá de su encuentro personal en Halle y de que colaboraron en el Jahrbuch, podemos encontrar referencias desperdigadas en sus obras, conferencias, algunas cartas y anotaciones que hicieron al leerse mutuamente ${ }^{8}$. De todas esas indicaciones, algo queda claro: existe un punto irreconciliable entre sus análisis fenomenológicos, aquel por el cual tenemos, de un lado, una fenomenología trascendental y, del otro, una ontología fenomenológica. En lo que sigue, intentaremos trazar algunas líneas generales de lo que sería un diálogo entre Husserl y Scheler sobre la objetividad de los valores esperando que el contraste entre sus modos de proceder aclare el fenómeno. Para ello, recordaremos, primero, los caminos que llevan a cada uno a sostener la objetividad de los valores; luego, intentaremos mostrar en qué radican sus coincidencias y desacuerdos.

Los caminos por los que Husserl y Scheler llegan a sostener la objetividad de los valores, aunque tienen una base común que quisiéramos mostrar, son, como las consecuencias éticas que de ahí extraen, distintos. En el caso de Husserl, hay que considerar el desarrollo de su concepción de la vida emocional y, en particular, la cuestión de la relación entre los actos objetivantes y no objetivantes. Como se sabe, en la quinta de las Investigaciones lógicas, Husserl discute el principio de Brentano según el cual los "fenómenos psíquicos" son representaciones o reposan sobre representaciones introduciendo los conceptos de materia y cualidad intencionales como momentos abstractos de la "esencia intencional" de los actos. Ello lo lleva a sostener, en primer lugar, que el sentir y el querer no son más que cualidades intencionales no objetivantes, es decir, solo pueden ser actos con pleno derecho, y necesariamente actos complejos, por una materia intencional que les presta la referencia objetiva a un qué y un cómo, pero que, a su vez, es siempre materia de una cualidad de acto objeti-

\footnotetext{
${ }^{8}$ Sobre el encuentro en Halle, cf. Scheler, Max, Die deutsche Philosophie der Gegenwart (1922), en Gesammelte Werke, vol. 7, Berna: Francke, 1973, p. 308. Indicaciones bibliográficas sobre referencias de Husserl a Scheler se encuentran en Melle, Ullrich, "Schelersche Motive in Husserls Freiburger Ethik", en Vom Umsturz der Werte in der modernen Gesellschaft, Bonn: Bouvier, 1997, pp. 203 ss. y nota 8. Cf. también "Les annotations dans le Formalisme de Max Scheler/Randbemerkungen zu Schelers Formalismus", ed. y trad. de Heinz Leonardy, en Études phénoménologiques, vol. VII, no 13-14 (1991), pp. 3-57.
} 
vante $^{9}$. En segundo lugar, la posibilidad de cumplimiento o plenificación de todo sentir y querer, aunque no se agota en la de los actos objetivantes, sí está condicionada por ella ${ }^{10}$, de modo que no podemos hablar en sentido estricto de validez del sentir y del querer entendidos como actos fundados no objetivantes. Finalmente, solo pueden ser expresados mediante un acto de reflexión que los hace objeto. Así, Husserl sostiene al final de la "Sexta investigación" que la expresión de los actos no objetivantes solo es un caso particular de los enunciados o expresiones de los actos objetivantes ${ }^{11}$.

Distintos autores han mostrado la dificultad de este análisis de la vida emocional y conativa ${ }^{12}$. Se trata de una consecuencia de dos aspectos correlativos. Primero, solo permite sostener una "teoría subjetivista" de valores ${ }^{13}$, pues si las determinaciones axiológicas y prácticas no pertenecen a la referencia al objeto, es decir, a la materia intencional, no se puede decir que el objeto sea bello, odiable o amable en sí mismo ${ }^{14}$. Así, de modo correlativo, el análisis de las Investigaciones excluye a los actos no objetivantes de la esfera de la razón, pues no siendo más que los momentos abstractos de cualidades intencionales no téticas, la validez del sentir y el querer no es posible sin el acto de identificación que da cumplimiento a la representación del objeto. Si no podemos hablar de

9 Cf. Husserl, Edmund, Logische Untersuchungen. Erster Band. Erster Teil. Untersuchungen zur Phänomenologie und Theorie der Erkenntnis, ed. Ursula Panzer, Dordrecht/Boston/Londres: Kluwer, Husserliana, volumen XIX/1, 1984, § 41, pp. 514-515 (en adelante, Hua XIX/1).

${ }^{10}$ Cf. Husserl, Edmund, Logische Untersuchungen. Zweiter Band. Zweiter Teil. Untersuchungen zur Phänomenologie und Theorie der Erkenntnis, ed. Ursula Panzer, Dordrecht/Boston/Londres: Kluwer, Husserliana, volumen XIX/2, 1984, § 13, p. 583 (en adelante, Hua XIX/2).

${ }^{11}$ Hua XIX/2, § 70, p. 749.

${ }^{12}$ Cf. Melle, Ullrich, "Objektivierende und nicht-objektivierende Akte", en IJsseling, S. (ed.), HusserlAusgabe und Husserl-Forschung, Dordrecht/Boston/Londres: Kluwer, 1990, pp. 35-49; Serrano de Haro, Agustín, "Actos básicos y actos fundados", en Anuario filosófico, vol. XXVIII, n 1 (1995), pp. 61-89; Benoist, Jocelyn, Les limites de l'intentionnalité. Recherches phénoménologiques et analytiques, París: Vrin, 2005, pp. 151-169; Bégout, Bruce, "Pulsion et intention. Husserl et l'intentionnalité pulsionnelle", en Goddard, Jean Christophe, La pulsion, París: Vrin, 2006, pp. 145-150.

13 Serrano de Haro, Agustín, op. cit., p. 72.

${ }^{14}$ Como se ha mostrado, Husserl parece dudar de su descripción en el § 15 de la "Quinta investigación". Interesado en establecer la unidad esencial de los actos, distingue entre sentimientos en tanto actos y sentimientos sensibles (sinnliche Gefühle). Los primeros, en acuerdo con la descripción de Brentano, están constituidos por dos intenciones: una fundante que presenta el objeto y otra, fundada, que da el objeto sentido, querido, deseado, etc. y que no puede existir sin la intención fundante. Ello no implica, sin embargo, integrar en su materia intencional algo así como determinaciones objetivas de los valores. Los sentimientos sensibles, también llamados "sensaciones sentimentales" o "afectivas" (Gefüh/sempfindungen), son un elemento no intencional y, por tanto, no esencial, de los actos. Entrelazadas con las sensaciones, son "animadas" por los caracteres intencionales, de modo que son condiciones para la constitución de los actos emocionales. Pese a que Husserl se refiere a la sensación de placer (Lustempfindung) como "aprehendida y localizada, de un lado, como excitación sentimental del sujeto psicofísico que siente $y$, del otro, en tanto propiedad objetiva: el suceso aparece como recubierto por un velo rosado" (Hua XIX/1, § 15b, p. 408), la coloración emocional o afectiva relativa a esa "propiedad objetiva" no corresponde a un valor, sino que es del orden de la representación. Las intenciones de los sentimientos en tanto actos vienen después y podrían desaparecer sin que se modifique el acto básico, sostiene Husserl. 
posición ni de intuición respecto del sentir y del querer, no podemos hablar de razón axiológica ni práctica ${ }^{15}$.

La situación cambia en las Ideas de 1913, donde la fenomenología ya no es una "psicología descriptiva" limitada al análisis de los "ingredientes reales" de las vivencias intencionales y ya no abandona el objeto a la esfera de lo trascendente. En tanto dado y potencialmente dado, el objeto intencional y todos sus rasgos a priori forman parte de la nueva inmanencia fenomenológica que admite en ella trascendencias. Esto no implica que Husserl renuncie al carácter fundado de la vida emocional y volitiva. Se mantiene una doble intencionalidad, la de los actos de la representación -sea la creencia originaria, una modalidad de creencia o una conciencia de neutralidad- y la de los fundados, de manera que, de modo correlativo, el objeto intencional tiene un sentido doble: la simple "cosa" (blosse "Sache") y el objeto intencional pleno que incluye el valor ${ }^{16}$. En relación con las Investigaciones, Husserl extiende el concepto de tesis y, en este sentido amplio, entiende el sentir y el querer como posiciones ${ }^{17}$. Con ellas, surgen nuevos estratos noemáticos $y$, así, junto con las apercepciones de cosas, tenemos apercepciones de la objetividad axiológica, noemas emocionales ${ }^{18}$. Los valores ya no son agregados de la reflexión ni se disuelven en una cualidad intencional, sino que reclaman su propia ontología, que remite al sentir y al querer entendidos como actos "objetivantes" y "originariamente constituyentes" ${ }^{19}$. A partir de esta generalización del concepto de tesis, Husserl puede, en las lecciones de ética que imparte en Gotinga en 1914, justificar el método de la analogía entre la ética y la lógica para refutar el escepticismo ético y fundar filosóficamente la ética a partir de una axiología y práctica formales. Si una teoría formal de los valores y una teoría formal de la práctica son posibles, es porque las leyes de las que se ocupan estas disciplinas, relativas a las estructuras esenciales de los valores y de los fines, remiten a sus versiones normativas co-

\footnotetext{
${ }^{15}$ Melle, Ullrich, "Objektivierende und nicht-objektivierende Akte", op. cit., p. 41.

${ }^{16}$ Husserl, Edmund, Ideen zu einer reinen Phänomenologie und phänomenologischen Philosophie, Erstes Buch, Allgemeine Einführung in die reine Phänomenologie, ed. Karl Schuhmann, La Haya: M. Nijhoff, Husserliana, volumen III/1, 1976, § 37, p. 76 (en adelante, Hua III/1).

${ }^{17}$ Ibid., § 117, p. 269.

18 "Der neuer Sinn bringt eine total neue Sinnesdimension herein, mit ihm konstituieren sich keine neuen Bestimmungsstücke der blossen 'Sachen', sondern Werte der Sachen, Wertheiten, bzw. konkrete Wertobjektitäten: Schönheit und Hässlichkeit, Güte und Schlechtigkeit; das Gebrauchsobjekt, das Kunstwerk, die Maschine, das Buch, die Handlung, die Tat usw." (ibid., § 116, p. 267).

${ }_{19}$ Ibid., $\S 117$, p. 272.
} 
rrespondientes, es decir, a normas sobre la validez de los actos valorativos del sentir y los actos prácticos de la voluntad.

Así, Husserl funda el paralelo entre la razón lógica, axiológica y práctica en la analogía, y por tanto también en la irreductibilidad, de las tres especies de $\operatorname{actos}^{20}$. Como la evidencia no es más exclusiva del pensar o del juzgar, la tarea de la ética consiste en mostrar los principios a priori a partir de los cuales el sentir y el querer en general son o no legítimos, justificados o correctos; y en esclarecer, según las conexiones esenciales posibles que permita ver la variación imaginativa, el entrelazamiento entre los tres tipos de actos. Para ser precisos, ya en Ideas II Husserl trabaja con el concepto de intuición axiológica (Wertnehmung), y como se ha mostrado, la cuestión se decide en las lecciones de ética de 1908/09 ${ }^{21}$. En varias ocasiones a lo largo de esas lecciones, Husserl duda sobre el sentido y la necesidad de mantener la distinción entre actos objetivantes y no objetivantes. Finalmente, opta por mantenerla señalando que los actos valorativos del sentir no se dirigen a los objetos de las representaciones que las fundan, sino a valores ${ }^{22}$, y sin renunciar a la idea unitaria de la razón: el sentido teleológico-normativo es un a priori que atraviesa todas las esferas de $\operatorname{actos}^{23}$. Ello significa que también a los actos valorativos y volitivos les pertenece la distinción entre intención y cumplimiento; también en ellos la evidencia es el telos que pone la norma, de modo que pueden ser intenciones vacías o plenas, claras o confusas, fundadas o devaluadas. Así pues, la legitimidad e ilegitimidad también pertenecen a la vida emocional; dicho de otro modo, la racionalidad es también axiológica y práctica. Esta convicción está a la base de la determinación del concepto de ética y de vida ética de las lecciones de Friburgo, cuyos análisis genéticos sobre el valor y el deber muestran que estos

20 Cf. Husserl, Edmund, Vorlesungen über Ethik und Wertlehre, 1908-1914, ed. Ullrich Melle, Husserliana, volumen XXVIII, Dordrecht/Boston/Londres: Kluwer, 1988, especialmente §§ 7-8, (en adelante, Hua XXVIII).

${ }^{21}$ Cf. Benoist, Jocelyn, Les limites de I'intentionnalité, op. cit., pp. 164 ss.

22 "...wertende Akte sind nicht auf Objekte 'gerichtet', sondern auf Werte. Wert ist nicht Seiendes, Wert ist etwas auf Sein oder Nicht-Sein Bezügliches, aber gehört in eine andere Dimension" (Hua XXVIII, § 12c, p. 340). Este pasaje marca una diferencia no solo respecto de las Investigaciones, donde los actos valorativos, en tanto no objetivantes, no poseen una referencia objetiva propia, sino también respecto de Ideas I, donde los valores se presentan como una nueva región del ser. Al respecto, Schuhmman sostiene que no hay contradicción entre las lecciones de 1908/09 y las Ideas I, porque "Werte sind sehr wohl Gegenstände (Etwasse), aber kein Naturseiendes" (Schuhmman, Karl, "Probleme der Husserlschen Wertlehre", en Philosophisches Jahrbuch, n 98 (1991), p. 109, nota 6).

23 "Vernunft ist ein Titel für das die betreffenden Aktsphären durchwaltende teleologische Apriori; teleologisch nenne ich es hier, weil es auf Verhältnisse der Richtigkeit und Unrichtigkeit geht und die Richtung auf Gegenstand und Wert jene Richtung im Sinne der Richtigkeit ist" (Hua XXVIII, § 12d, p. 343). 
tienen su fuente última en el juego entre la motivación activa y pasiva que es también un ámbito de espiritualidad emocional.

Recordemos ahora la estrategia scheleriana para sostener la objetividad de los valores. Como sabemos, de ello se ocupa Scheler desde la primera sección del Formalismus, cuya estructura está trazada por ocho críticas que dirige a la ética kantiana ${ }^{24}$. Apoyándose en el concepto husserliano de a priori material que Scheler tratará explícitamente en la segunda sección del libro, la primera crítica ataca el supuesto kantiano según el cual toda ética material ha de ser una ética de bienes o de fines. La primera tarea es, pues, distinguir los valores de los bienes o "cosas de valor" (Wertdinge). Para ello, Scheler realiza una serie de análisis que se pueden dividir en dos grupos. El primero busca mostrar que los valores no son los términos que designan lo que solemos llamar "propiedades" comunes de las cosas o de los seres humanos. Sostener eso sería confundir los valores con sus portadores o depositarios (Trägern), como cuando nos referimos al modo peculiar en que una fruta es agradable al gusto por las sensaciones que genera o por las propiedades que captamos al percibirla. El segundo grupo de análisis está destinado a mostrar la independencia del ser de los valores respecto de sus portadores. Para ello, plantea una serie de casos en los que el valor de algo o alguien nos es dado sin que hayamos identificado a su portador o sin que sepamos por qué captamos en él dicho valor: "por ejemplo dice Scheler- un hombre nos resulta desagradable y repulsivo, o agradable y simpático, sin que podamos indicar en qué reside eso [... $]^{\prime 25}$ o como cuando descubrimos el valor de la belleza en un objeto cuyo significado desconocemos. En términos de Husserl, los actos de la representación pueden ser simples menciones sin que ello impida la plenitud intuitiva del valor dado en un acto emocional. Y ese es precisamente el problema que se ha de discutir, anota Husserl en su ejemplar del Formalismo ${ }^{26}$. Más aún, Scheler sugiere que comprendemos el significado de un objeto gracias a su valor, que funciona de "mensajero de su peculiar naturaleza"27. Ello no significa, sin embargo, que los

\footnotetext{
${ }^{24}$ Cf. Scheler, Max, Der Formalismus in der Ethik und die materiale Wertethik. Neuer Versuch der Grundlegung einer ethischen Personalismus, edición de Mandfred Frings, Gesammelte Werke, tomo 2, Bonn: Bouvier, 2000, pp. 30-31 (en adelante, GW 2); Ética, introducción y edición de Juan Miguel Palacios, traducción de Hilario Rodríguez Sanz, Madrid: Caparrós, 2001, pp. 48-49.

${ }^{25}$ GW 2, p. 40; Ética, p. 63.

26 "Les annotations dans le Formalisme de Max Scheler/Randbemerkungen zu Schelers Formalismus", op. cit., pp. 25 y 27.

${ }^{27}$ GW 2 p. 40 ; Ética, p. 64.
} 
bienes se funden sobre las cosas en el sentido de que, para que algo sea un bien, debe ser antes una cosa. En realidad, Scheler distingue entre la cosa (Ding), el bien (Gut) y el objeto (Sache), y sostiene que, en la intuición natural del mundo -correspondiente a un "orden genético" relativo a la estructura orgánica del ser viviente del caso-, lo primero que nos es dado es un objeto (Sache), entendido como una cosa natural cuya experiencia, fundada en un valor que no le es esencial sino fortuito, implica la referencia a un poder disponer de ella a nuestra voluntad. Solo después, si hacemos abstracción de los valores, nos es dada una cosa o un bien si hacemos abstracción de la naturaleza de la $\operatorname{cosa}^{28}$. Así pues, Scheler afirma que los bienes son a las cualidades de valor lo que las cosas son a las cualidades, porque encuentra como rasgo esencial de los bienes que, en ellos, un determinado valor guía y estructura la aprehensión tanto de sus otras cualidades axiológicas como de aquellas del color o la forma ${ }^{29}$.

El objetivo de Scheler con esta caracterización de los bienes es mostrar que, dado que se fundan en los valores, podemos rechazar, por contingente, una ética material de bienes sin que ello implique rechazar, por la misma razón, una ética material de valores. El mismo argumento vale para las éticas de fines, lo que nos ubica en el ámbito de la vida conativa. Scheler distingue entre las metas o los objetivos de la tendencia (Strebensziel) y los fines de la voluntad (Willenszweck). En los primeros, descubre dos componentes esenciales: uno de imagen (Bildkomponent) y otro, de valor (Wertkomponent). Como en el caso de los bienes, es este último el que cumple el rol director: la imagen de la meta puede ser borrosa o estar ausente, mientras que su valor es claramente vivido. La orientación y el desarrollo de los componentes imaginativos dependen de la donación del valor sentido en la meta a la que tendemos o aspiramos. Ello se debe a que, entre el sentir y el tender, existe una relación de "motivación práctica": "en cualquier tendencia hacia algo va incluso el [sentir] de algún valor, que fundamenta el componente de imagen o significación de la tendencia" $^{\prime 30}$. El sentir intencional tiene, para Scheler, una "función cognitiva"31: en su

\footnotetext{
${ }^{28}$ Ibid., p. 44; ibid., p. 68.

${ }^{29}$ Ibid.

30 "In jegliches Streben nach etwas geht (...) ein Fühlen irgendeines Wertes, die Bild- oder Bedeutungskomponente des Strebens fundierend, ein. Dieses eigenartige Verhältnis ist jenes, das gemeinhin als praktische Motivation bezeichnet wird" (ibid., p. 346; ibid., p. 465).

${ }_{31}$ Ibid., p. 263, nota; ibid., pp. 360-361, nota 19.
} 
realización, el mundo de objetos se nos muestra originariamente, pero solo en su aspecto axiológico ${ }^{32}$. Entendido como un modo primario de intencionalidad, cuyo correlato son los valores en tanto "clase propia de objetos", todo sentir necesariamente implica la posibilidad de plenificación ${ }^{33}$. Y así no solo no necesitan de la mediación de los actos objetivantes de la representación o el juicio, como sostienen Brentano y Husserl, sino que ellos mismos pueden ser considerados como objetivantes ${ }^{34}$. La representación solo es necesaria para los correlatos del querer. La voluntad solo puede proponerse fines si se llega a formar, gracias nuevamente a la vida emocional, al sentir intencional, la representación del componente imaginativo de la meta de la tendencia, y si ella es dada a nuestro querer como algo que se ha de realizar, que debe ser real (realseinsoIlender). A ello hay que agregar que los fines de la voluntad solo surgen a partir de la elección entre las metas de las tendencias, elección que, a su vez, se funda en la preferencia y subordinación, actos por los que conocemos las relaciones de superioridad o inferioridad de los valores. Así, el cambio de un mundo de bienes o de fines no implica la relatividad de los valores, sino solo la historicidad de las reglas de preferencias que dominan una época, el ethos. La ética entonces debe ocuparse de las esencias y relaciones esenciales entre los valores y los estratos de la vida emocional.

Llegados a este punto, parece claro que el desacuerdo entre Husserl y Scheler se encuentra en el orden de fundación de los actos. Pero analicemos primero el acuerdo. Este radica en la distinción entre dos planos: el ser esencial o ideal de los valores y su existencia efectiva. En los bienes, dice Scheler, los valores devienen reales ("wirklich"): "[...] en el bien es objetivo el valor (siempre lo es) $y$ al mismo tiempo real. Con cada nuevo bien se produce un verdadero aumento de valor en el mundo real. Por el contrario, las cualidades valiosas son 'objetos ideales', como lo son los olores y las cualidades de sonido" ${ }^{35}$. De

\footnotetext{
32 Ibid., p. 265; ibid., p. 363.

33 Ibid., p. 263; ibid., p. 361.

34 "Gerade das häufige Fehlen von Bildobjekten im intentionalen Fühlen zeigt, dass das Fühlen seinerseits von Hause aus ein 'objektivierender Akt' ist, der keiner Vorstellung als Vermittlers bedarf" (ibid., p. 265; ibid., p. 363).

35 Ibid., p. 43 ; ibid., p. 67.
} 
modo semejante, Husserl distingue "valores reales", en tanto bienes temporales que desaparecen con la aprehensión del valor, y "valores ideales" supratemporales ${ }^{36}$. La objetividad axiológica radica, entonces, para ambos autores, en el ser esencial o ideal de los valores, no en su existencia efectiva que depende, más bien, del carácter empírico de las cosas, situaciones o personas valiosas, en términos de Scheler, de los portadores contingentes. Así, los fenomenólogos coinciden en la objetividad de los valores porque los tratan como objetividades ideales (Husserl) o como esencias (Scheler). En este sentido, Husserl afirma que el objetivismo que él defiende en la axiología significa idealismo ${ }^{37}$ y Scheler sostiene que es necesario partir de la idea del valor, pues ella es indiferente a la esfera de la existencia y del deber ${ }^{38}$. Además, así como las esencias solo son dadas en la efectuación propia de intuiciones eidéticas, a los valores solo accedemos en la realización misma de vivencias y actos emocionales $^{39}$, lo cual no significa que su ser se agote en ellos ${ }^{40}$, sino solo que son, como las esencias, indefinibles. Los mostramos o los vivimos, no los definimos teóricamente. "La teoría no conduce a valores", dice Husserl en sus lecciones de $1908 / 9^{41}$.

De otro lado, hay que señalar que este concepto de objetividad no implica negar la individualidad personal. Se podría pensar que esto solo es posible para el personalismo ético de Scheler y sus conceptos de ethos y ordo amoris, de los que no nos podemos ocupar aquí. Sin embargo, insatisfecho de las normas éticas planteadas en Gotinga, Husserl planteará un nuevo concepto de valores que coincide con el concepto scheleriano de "lo bueno en sí para mí". Es "para mí", dice Scheler, porque el valor incluye una referencia vivida a una persona individual, que funda luego deberes individuales a los que uno se siente "llamado"42. Pero es "en sí" porque, como todos los valores, es independiente de mi saber,

\footnotetext{
36 "Es gibt zwar Werte, die realen Werte, reale Güter jeder Art, die zeitliche Ereignisse sind und die im Wertnehmen sich aufbrauchen, mit inm vorübergehen wie das Konzert mit dem Geniessen des Konzerts, aber es gibt auch ideale Werte, nämlich überzeitliche" (Hua XXXVII, § 16, p. 72). Más adelante, Husserl emplea también la expresión "valor concreto" para referirse "al objeto en su valor" (ibid., p. 75 y $\S 17$, p. 79). Cf. también Erste Philosophie. Zweiter Teil: Theorie der phänomenologischen Reduktion, ed. Rudolf Boehm, Husserliana, vol. VIII, La Haya: M. Nijhoff, 1959, p. 104 (en adelante, Hua VIII).

${ }^{37}$ Hua XXVIII, § 11 b, p. 89.

38 GW 2, p. 218; Ética, p. 303.

${ }^{39}$ Cf., por ejemplo, ibid., p. 87; ibid., p. 127.

${ }^{40}$ Hua XXXVII, § 15, p. 69, § 16, p. 73; Hua XXVIII, § 10, p. 80, § 11b, p. 88, § 3, pp. 249-250.

${ }^{41}$ Hua XXVIII, § 5d, p. 268; cf. también § 7a, p. 277: "...ohne Wertung kein Wert, das heißt, dass Werte in gewisser Weise im Werten bewusst werden und nur im Werten gegeben sein können etwa so, wie Ding nichts für unș sein könnte ohne Wahrnehmen und ohne Wahrnehmen nie gegeben sein könnte".

${ }^{42}$ GW 2, p. 482; Ética, p. 637.
} 
de modo que el carácter individual no elimina su objetividad. Desde 1916/17, después de haber defendido, en sus lecciones de 1914, normas de la preferencia y un imperativo categórico universal inspirados en Brentano, Husserl empieza a distinguir, en sus manuscritos de investigación, entre valores que son solo objetivos, en el sentido de intersubjetivos, y valores que además son subjetivos o individuales porque tienen como fuente al amor ${ }^{43}$. A ellos se referirá más tarde, en las lecciones de Introducción a la ética de Friburgo, como "valores de $\operatorname{amor}^{\prime 44} \mathrm{y}$, en uno de los artículos preparados para The Kaizo, los relacionará estrechamente con la personalidad, al punto que uno no puede sino "amarlos dice Husserl- desde el centro más íntimo de mi persona -'con toda mi alma'-: amarlos como los míos, como aquellos a los que yo mismo, tal como soy, pertenezco inseparablemente ${ }^{\prime 45}$. Es por esta idea de valores objetivos pero personales que Husserl planteará un imperativo categórico individual ${ }^{46}$ y que Scheler, quien no cree que la ética fenomenológica deba ser imperativa, sostiene que esta "no puede ni debe sustituir a la conciencia moral del individuo"47.

Regresemos ahora al desacuerdo respecto del orden de fundamentación de actos. Creemos que su origen radica en que Scheler distingue un orden de la donación de un orden óntico. En el primero, prima el valor sobre el ser: "las cualidades de valor y las unidades de valor pertenecientes a este orden, están dadas previamente a todo lo que pertenece al estrato del ser ajeno a los valores: de manera que ningún ente totalmente libre de valor puede 'hacerse originariamente' objeto de una percepción, recuerdo, esperanza, y en segundo lugar, del pensamiento y juicio, sin que su cualidad de valor o su relación de valor nos haya sido dada de algún modo de antemano frente a otra cosa (igualdad, diferencia, etc.) $[\ldots]^{\prime \prime 48}$. De este primado no debe hacerse una hipóstasis,

\footnotetext{
43 Sobre este tema, cf. Melle, Ullrich, "Husserls personalistische Ethik", en Centi, Beatrice y Gianna Gigliotti (eds.), Fenomenologia della ragion pratica. L'ética di Edmund Husserl, Nápoles: Bibliopolis, 2004, especialmente, pp. 347-352.

${ }^{44}$ Hua XXXVII, anexo XII, p. 339.

45 Renovación del hombre y de la cultura, intr. de Guillermo Hoyos, trad. de Agustín Serrano de Haro, Barcelona/Iztapalapa, México: Anthropos/Universidad Autónoma Metropolitana, 2002, p. 30; Aufsätze und Vorträge (1922-1937), ed. T. Nenon y H.R. Sepp, Husserliana, vol. XXVII, Dordrecht/Boston/Londres: Kluwer, 1989, p. 28 (en adelante, Hua XXVII).

${ }^{46}$ Ibid., p. 43; ibid., p. 41. Cf. el § 6 de "Wert des Lebens. Wert der Welt. Sittlichkeit (Tugend) und Glückseligkeit <Februar 1923>", ed. Ullrich Melle, en Husserl Studies, vol. 13, n 3 (1996-1997), pp. 201-235; "Valor de la vida. Valor del mundo. Moralidad (virtud) y felicidad <Febrero de 1923>", trad. de Julia V. Iribarne, en: Acta Fenomenológica Latinoamericana, vol. III, Lima/Morelia (México): PUCP/Universidad Michoacana de San Nicolás Hidalgo, 2009, pp. 810 ss.

${ }^{47}$ GW 2, p. 486; Ética, p. 642.

${ }^{48}$ Scheler, Max, La esencia de la filosofía y la condición moral del conocer filosófico, trad. de Elsa Tabernig, Buenos Aires: Editorial Nova, $1966^{3}$, p. 31 (en adelante, EF); "Vom Wesen der Philosophie und der
} 
pues en el orden óntico los valores son secundarios: "También aquí 'lo posterior en sí puede ser 'lo anterior para nosotros', que era lo que afirmaba Aristóteles como regla general referente a la relación del conocer y el ser. La afirmación de Aristóteles no solo es válida, sino que debe serlo, puesto que es una proposición evidente la de que a todas las cualidades 'corresponde' un ser subsistente, al cual son inherentes esas cualidades -aunque las mismas puedan darse de forma aislada de sus portadores y estén siempre sometidas por esencia a un orden propio fundado en su contenido" ${ }^{49}$.

Así pues, desde el punto de vista scheleriano, habría que decir que si Husserl afirma, en las lecciones de ética de Friburgo, que "[d]onde no existe una donación previa originariamente sensible, el sentimiento y la tendencia no pueden hablar, ahí tampoco se puede valorar ni hacer configuraciones prácticas" ${ }^{\prime 50}$, es porque confunde el orden de la donación con el orden óntico poniendo como fundante en el primero lo que solo es tal en el segundo. Para Scheler, todo ser libre de valor (wertfrei) es una abstracción, un producto artificial de un acto intelectual, y considerarlo como originario no solo en tanto existente, sino también en tanto dado, es deformar la cosa misma ${ }^{51}$. A primera vista, esta crítica parece alcanzar el proceder genético de la deconstrucción ( $A b b a u$ ) que Husserl sigue en el "Excurso" de las lecciones de ética de Friburgo; sin embargo, ese desmontaje de los estratos de sentido del mundo no busca sostener que en la actitud natural tenemos que ver con seres indiferentes a los valores y que solo posteriormente se los atribuimos. "Todo lo que es -dice Husserl- toca el sentimiento, todo ente es apercibido en apercepciones de valor y así despierta tomas de posición que desean, no plenificadas o plenificadas ${ }^{\prime 52}$. El ser o el mundo libre de valor también es para Husserl una abstracción ${ }^{53}$. Precisamente, de ella se sirve para mostrar, desde el punto de vista de la fenomenología genética, que nuestra experiencia natural del mundo es, desde el inicio y de modo inmediato, emocional, valorativa y práctica. El carácter inmediato de la experiencia de valores efectivamente existentes no está en cuestión.

moralischen Bedingung des philosophischen Erkennens", en: Vom Ewigen im Menschen, Gesammelte Werke, vol 5, Berna: Francke, 1954, p. 80 (en adelante, GW 5).

${ }^{49} E F$, p. 34; GW 5, p. 82.

50 Hua XXXVII, § 31, p. 146

${ }^{51} E F$, p. $31 ;$ GW 5, p. 82.

52 Husserl, Edmund, Zur Phänomenologie der Intersubjektivität. Texte aus dem Nachlass, Dritter Teil : 1929-1935, ed. Iso Kern, Husserliana, volume XV, The Hague: M. Nijhoff, 1973, pp. 404-405.

53 Hua XXXVII, "Exkurs", § 9, p. 295. 
Si miramos las cosas desde el punto de vista noético, hay que decir que mientras Husserl insiste en el entrelazamiento de los tipos de actos, tanto en las lecciones de Gotinga como en las de Friburgo ${ }^{54}$, Scheler está sobre todo interesado en la independencia de los actos emocionales respecto de los otros tipos de actos. Ello le vale, en efecto, la crítica de Strasser, para quien la distinción entre cosas y propiedades que solo son dadas en la percepción y el entendimiento, de un lado, y valores que solo son dados en el sentir, de otro, es insostenible por artificial $^{55}$. Ciertamente, Husserl entiende el entrelazamiento de los tipos de actos mediante la fundación del querer en el sentir y de este último en los actos de la representación. Pero cuando sobre una percepción se funda una intención valorativa, es decir, cuando el interés es axiológico y no teórico, la percepción no es más que un acto parcial, subordinado al acto global. No hace más que servir al sentir o a la voluntad que lo atraviesa ${ }^{56}$.

Se podría añadir que si Husserl se ubica en el orden óntico, se debe a que en la actitud natural no aprehendemos los valores, como dice Scheler, en tanto "cualidades objetivas puras" 57 independientes de sus portadores, sino a partir de ellos. Por supuesto, hablamos de la justicia y de la belleza en general, y nos entendemos. Scheler tiene razón cuando sostiene que accedemos a los valores sin preguntarnos por su existencia efectiva y sin necesidad de representárnoslos como propiedades de sus portadores ${ }^{58}$. Pero cuando esto ocurre, hemos pasado de una actitud axiológica a una teórica ${ }^{59}$. Falta aquello que es tan importante para Scheler, la efectuación (Vollzug) del acto emocional mismo, o bien ha pasado a un segundo plano. Por eso, cuando considera el punto de vista de la actitud natural, Scheler afirma que primero nos son dados los bienes y "[s]olo en segundo término nos son dados los valores que sentimos en esos bienes $[\ldots]^{\prime \prime 60}$.

Esclarecida la cuestión del orden de la fundamentación de los actos, queda en pie un sentido más profundo de fundamentación fenomenológica que separa

\footnotetext{
${ }^{54}$ Cf., por ejemplo, Hua XXVIII, § 7, p. 65 y Hua XXXVII, § 45, p. 229.

55 Strasser, S., Das Gemüt. Grundgedanken zu einer phänomenologische Philosophie und Theorie des menschlichen Gefüh/slebens, Utrecht/Anveres/Friburgo: Herder, 1956, p. 19. Sobre el tema del orden de fundamentación, cf. el excelente artículo de Fernández Beites, "Cosas, valores y tendencias. Husserl frente a Scheler", en Escritos de filosofía, n 44 (2004), pp. 163-190, en el que defiende la posición husserliana.

${ }^{56}$ Cf. Hua VIII, pp. 101 y 104.

${ }^{57}$ GW 2, pp. 39-40; Ética, p. 63.

58 Ibid., p. 35 ; ibid., p. 57.

${ }^{59}$ Hemos "modificado nuestro interés" (cf. Hua VIII, p. 98-106).

60 GW 2, p. 78; Ética, p. 117.
} 
a Husserl de Scheler y que nos recuerda no solo que en una "disputa fenomenológica" cada uno es responsable de qué muestra y de cómo lo muestra, sino también del acto libre con el que comienza a filosofar, es decir, de la efectuación apropiada de la reducción fenomenológica. Para Husserl, esta es primero trascendental y, luego, eidética; en el tema que nos ocupa, eso implica hablar de las "operaciones constitutivas de valor" por parte del sentir en tanto que valorar ${ }^{61}$, implica hablar "de la unificación sintética de las intenciones de valor que se plenifican continuamente unas en otras ${ }^{\prime \prime 2}$. Para Scheler, en cambio, la reducción conduce directamente al aspecto esencial, no existencial ni real, del ser, lo que implica que los valores ni se crean ni se destruyen ${ }^{63}$, solo se descubren en nuestras vivencias emocionales $y$, a partir de ellos, nos limitamos a seleccionar por "funcionalización" lo que será destacado para entrar a la esfera de lo dado.

Así pues, desde el punto de vista de Husserl, la reducción scheleriana es eidética ${ }^{64}$, porque no comprende el sentido de su reformulación de la revolución copernicana de $\mathrm{Kant}^{65} \mathrm{y}$, entonces, permanece en el aspecto objetivo de la correlación, en una metafísica ingenua que justifica con el concepto de a priori material que encuentra en las Investigaciones ${ }^{66}$. Desde el punto de vista de Scheler, la reducción husserliana es insuficiente: no basta suspender el juicio sobre el sentido y validez del mundo, es necesario también suspender las vivencias conativas y los actos prácticos que nos dan el carácter de realidad. Por eso, sostiene que en el conocimiento filosófico participa "el hombre entero" ${ }^{17} \mathrm{y}$ condiciona ese conocer a la efectuación de tres actos morales básicos: "1. El amor de la persona espiritual al valor y al ser absolutos; 2. la humillación del yo y del ego natural; 3. el autodominio [... $]^{\prime \prime 68}$. Esta es, pues, para terminar, la

\footnotetext{
${ }^{61}$ Hua XXXVII, § 37, p. 181; cf. también § 38, p. 192 y el anexo III, p. 326: "Wie die Sinnesdaten das Material sind für das Erlebnis, das wir Wahrnehmungserlebnis von Dingen mit den ihnen immanenten Dingerscheinungen nennen, so sind die sinnlichen Gefühle das Material für unsere Wertapperzeption, für die Erlebnisse, in denen wir etwa eine Melodie oder Symphonie, ein Gedicht usw. wertend erfassen und genießen in der Einheit eines synthetischen Gefüh/s".

62 Hua VIII, p. 104.

${ }^{63}$ GW 2, p. 266; Ética, p. 365.

64 "Les annotations dans le Formalisme de Max Scheler/Randbemerkungen zu Schelers Formalismus", op. cit., pp. 36 y 37.

${ }^{65}$ Ibid., pp. 34-37.

${ }^{66}$ Husserl, Edmund, "Phénoménologie et anthropologie", trad. de Didier Frank, en Notes sur Heidegger, París: Les Éditions de Minuit, 1993, p. 72. Cf. Hua XXVII, pp. 164-181.

${ }^{67}$ GW 5, p. 84 ss.; $E F$, p. 36 ss.

${ }^{68}$ Ibid., p. 89; $E F$, p. 45. A propósito del sentido ético de la reducción, cf. Rizo-Patrón, Rosemary, La agonía de la razón. Reflexiones desde la fenomenología práctica (en proceso).
} 
razón de que la primera evidencia en Husserl sea el yo trascendental y, en Scheler, que "algo es absolutamente" o que la "nada no es"69.

${ }^{69}$ GW 5, p. 93; EF, p. 50.

Investigaciones Fenomenológicas, vol. Monográfico 4/II (2013): Razón y Vida. 